\title{
BESPRECHUNGSAUFSATZ
}

\section{Verfassungsgerichtliche Kontrolle in Lateinamerika ${ }^{1}$}

\author{
Von Jürgen Samtleben, Hamburg
}

Das vorliegende Buch über die Verfassungsgerichtsbarkeit in Lateinamerika weckt mit seinem beeindruckenden Umfang und seiner weit gespannten Zielsetzung hohe Erwartungen. Der Autor, fünf Jahre lang als Auslandsmitarbeiter der Konrad-Adenauer-Stiftung in deren Rechtsstaatsprogramm in Lateinamerika tätig, hat für seine Doktorarbeit alles gesammelt, was er in diesem Zusammenhang zu Gesicht bekam. Dementsprechend werden in ausführlichen Berichten für jeden der 20 behandelten lateinamerikanischen Staaten die Probleme der Verfassungsgerichtsbarkeit historisch, rechtssystematisch und in praktischer Hinsicht geschildert. Leider wurde die Arbeit anschließend nicht von unnötigem Ballast befreit und einer wirklich stringenten Gliederung unterworfen. Dass die Darstellung mit Mexiko beginnt und anschließend die zentralamerikanischen Staaten zu einer einheitlichen Gruppe zusammenfasst, erscheint unter historischen Gesichtspunkten durchaus plausibel. Ähnliches mag für die karibischen Staaten gelten. Dagegen kann die folgende Ordnung der südamerikanischen Staaten nach ihrer strikt alphabetischen Reihenfolge nur als Verlegenheitslösung bezeichnet werden. Vorangestellt ist eine etwas kursorische Einleitung und ein gedrängtes 1. Kapitel, das für Lateinamerika den Einfluss der amerikanischen Verfassung im 19. Jahrhundert sowie das Vorbild des deutschen und spanischen Verfassungsgerichts im 20. Jahrhundert hervorhebt, aber keine begriffliche Klärung bringt. Insbesondere die unterschiedlichen Aspekte der Normenkontrolle, die sich in den Begriffspaaren ,vorbeugend - nachträglich“, ,diffus - konzentriert“, ,,abstrakt - konkret“, ,,allgemein - spezialisiert“, ,inter partes - erga omnes“, ,,ex nunc - ex tunc“ spiegeln, werden nicht als solche vorgestellt, vielmehr werden diese Termini in den folgenden Berichten ganz unreflektiert verwendet. Das zeigt sich z.B. an der unscharfen Gegenüberstellung der ,,abstrakten“ und der ,diffusen“ Normenkontrolle ${ }^{2}$.

1 Eine Kritik von Norbert Lösing, Die Verfassungsgerichtsbarkeit in Lateinamerika. Studien und Materialien zur Verfassungsgerichtsbarkeit, Band 83, Nomos Verlagsgesellschaft, Baden-Baden, 2001, 451 S., $€ 66,00$

2

Vgl. zu den verschiedenen Arten der Verfassungskontrolle die grundlegende Studie von Cappelletti, Judicial Review in the Contemporary World, Indianapolis, 1971. 
Im 2. Kapitel wendet sich der Autor zunächst der geschichtlichen Entwicklung in Mexiko zu. Weit ausholend und detailfreudig wird hier die mexikanische Geschichte und Verfassungsgeschichte von den Azteken bis zur Justizreform von 1994 nacherzählt, nur wenig untergliedert (manche Absätze gehen über mehrere Seiten) und nicht frei von Ungereimtheiten: Die „Acta del Ayuntamiento“ von 1808 war ein Akt der Ratsversammlung, aber keine „Akte des Rathauses“ (S. 42). Der Plan von Iguala von 1821 (nicht: 1921) wird zu Unrecht in die Kontinuität des vorangegangenen Unabhängigkeitskampfes gestellt (ebd.). Auch die „Definition des mexikanischen Staates“ als Bezeichnung der Zeit von 1822-1847 ist offenbar ein Übersetzungsfehler (S. 43). Und welchen Erkenntnisgewinn bringt es für den vorliegenden Zusammenhang, mit welchem Titel sich der mexikanische Diktator Santa Ana (durchgehend als Santa Anna bezeichnet) anreden ließ (S. 47)? Eingestreut in diesen Text finden sich erste Hinweise auf frühe Formen der Verfassungskontrolle und die Entstehung des für Mexiko typischen amparo-Prozesses, dessen Verständnis hier aber eigentlich schon vorausgesetzt wird. Nicht viel übersichtlicher ist der anschließende systematische Teil, der mit einer Beschreibung von Struktur und Organisation des Obersten Gerichtshofs beginnt und sich dann den einzelnen Verfahren zuwendet. Dem Autor ist es nicht gelungen, die verwickelte gesetzliche Regelung einigermaßen lesefreundlich zu vermitteln, was durch die ausführliche und z.T. duplizierte Wiedergabe der spanischen Gesetzestexte in den Fußnoten nicht kompensiert wird. Auch durch die (vom Computer erleichterte) ständige Wiederholung der vollständigen Schrifttumszitate wird der Fußnotenapparat unnötig aufgebläht. Die einzige in Fn. 170 zitierte Entscheidung ist an der angegebenen Stelle nicht auffindbar.

Wichtig ist die Feststellung, dass der Oberste Gerichtshof seit den Reformen von 1988 und 1994 auf die Prüfung von Verfassungsfragen beschränkt und die Legalitätskontrolle den nachgeordneten Gerichten zugewiesen ist (S. 51 f., 55). Dass bei den an erster Stelle dargestellten Verfassungskonflikten der Oberste Gerichtshof auch über die Gesetzmäßigkeit entscheidet, hätte daher der Erläuterung bedurft. Die anschließend behandelte abstrakte Normenkontrolle soll als neu geschaffenes Rechtsinstitut einen Ausgleich für die beschränkte inter-partes-Wirkung des amparo-Verfahrens bilden, wäre also nach dem folgenden Abschnitt über den amparo contra leyes besser verständlich. In diesem Abschnitt sind wiederum die Ausführungen über den recurso de inconstitucionalidad kaum zu verstehen, wenn man nicht zuvor die weiteren Erläuterungen über den amparo de casación gelesen hat - dem als Allheil(Rechts-)mittel gegen Urteile ohnehin von den verschiedenen Formen des amparo praktisch die größte Bedeutung zukommt. Erst dann wird deutlich, dass die amparo-Beschwerde gegen Urteile zwar unmittelbar und in „einziger Instanz“ von den höherinstanzlichen Kollegialgerichten entschieden wird, dass aber im Fall der inzidenten Normenkontrolle auch hier in „letzter Instanz“ eine Überprüfung der Verfassungsmäßigkeit durch den Obersten Gerichtshof stattfinden kann. Diese Möglichkeit ist allerdings durch die letzte Änderung des Art. 107 Nr. IX der Verfassung (Diario Oficial vom 
11.6.1999) erheblich beschränkt und dem Obersten Gerichtshof ein Ermessensspielraum eingeräumt worden, was der Text noch nicht berücksichtigt.

Im anschließenden 3. Kapitel, das der Verfassungsgerichtsbarkeit in Zentralamerika und Panama gewidmet ist, findet sich auf über 100 Seiten ein reiches Material ausgebreitet. Nach einem Vorspann über die Bestrebungen zur Bildung einer Zentralamerikanischen Union werden für jedes der mittelamerikanischen Länder eingehend dessen Verfassungsgeschichte, die Zusammensetzung des Obersten Gerichtshofs bzw. des Verfassungssenats und die verschiedenen Formen der Verfassungskontrolle dargestellt. Ein eigenes Verfassungsgericht besitzt in Zentralamerika nur Guatemala, was auch auf die Mitwirkung deutscher Experten bei der Ausarbeitung der Verfassung von 1985 zurückzuführen ist. Dass der Autor diesen Umstand überhaupt nicht erwähnt, ist umso erstaunlicher, als diese Mitwirkung gerade von der Konrad-Adenauer-Stiftung organisiert wurde ${ }^{3}$. Der Verfassungssenat am Obersten Gerichtshof von El Salvador (1983) wird zutreffend als Vorbild für Costa Rica (1989) und Nicaragua (1995) genannt, die entsprechende Regelung in Honduras (1996) aber übersehen ${ }^{4}$. Im Übrigen leidet die Darstellung darunter, dass trotz der Zusammenfassung in einem Kapitel die Möglichkeit zur einheitlichen Präsentation kaum genutzt wird; es macht vielmehr den Eindruck, als sei jeder der Länderberichte völlig selbstständig aufgrund des gerade für das jeweilige Land verfügbaren Materials bearbeitet worden ${ }^{5}$. So wird z.B. als Ausgangspunkt der Entwicklung die Verfassung von Cádiz von 1812 im Vorspann nur kurz erwähnt, in den nachfolgenden Berichten dagegen ganz unterschiedlich gewichtet ${ }^{6}$. Vor allem aber werden die verschiedenen Formen der Verfassungsklagen in den einzelnen Berichten immer wieder anders geordnet, die abstrakte und konkrete Nor-

Eingehend dazu Karpen, JöR 36 (1987) 527-555 mit dt.Übersetzung des Verfassungstextes, ebd. 555-596.

Corte Suprema de Justicia, Acta 61 vom 15.12.1995, Nr. 5, Gaceta Judicial 1287 (1996-97), 173 zur Einrichtung der „Sala de lo Constitucional Ad-Hoc“ ab 27.1.1996 (im Vorgriff auf eine gesetzliche Regelung).

Tatsächlich ist der Länderbericht Costa Rica bereits früher in dieser Zeitschrift erschienen, VRÜ 28 (1995), 166-192. Neueres Material ist daher nicht berücksichtigt; siehe etwa Solano Carrera, La aplicación directa de la Constitución (El caso de Costa Rica), Revista Judicial 61 (1995), 35-71; Bertolini / Fernández, La jurisdicción constitucional y su influencia en el Estado de Derecho, San José, 1996; Wilson / Handberg, From Judicial Passsivity to Judicial Activism: Explaining the Change within Costa Rica's Supreme Court, NAFTA L.Rev. 5 (1999), 522-543; Rueda Leal, Die verfassungsgerichtliche Unvereinbarerklärung verfassungswidriger Gesetze, 1999, für Costa Rica S. 115-170. Es fehlt aber auch die vorausgegangene Studie von Barker, Taking Constitutionalism Seriously: Costa Rica's Sala Cuarta, Florida J.Int.L. 6 (1990/91), 349-397.

Im Vorspann wird die aktive Beteiligung der zentralamerikanischen Abgeordneten (insbesondere aus Guatemala) bei den Beratungen in Cádiz betont (Fn. 227, 235), im Länderbericht Costa Rica das Kontrollsystem der Verfassung von Cádiz dargestellt (S. 84 f.) und schließlich im Länderbericht Guatemala deren genaue zeitliche Geltung präzisiert (Fn. 410). In den übrigen Berichten wird auf diese Verfassung nur kurz oder gar nicht hingewiesen; ausführlicher dazu Fuchs, Die Verfassungsentwicklung in Nicaragua, JöR 37 (1988), 621, 630 ff. 
menkontrolle teils getrennt, teils gemeinsam, die präventive Normenkontrolle teils vorweg, teils danach, die Grundrechtsschutzverfahren des habeas corpus und amparo teils am Anfang, teils am Schluss und in umgekehrter Reihenfolge behandelt. Der Leser, der die verschiedenen Rechtsordnungen miteinander vergleichen möchte, muss sich das Material dafür erst aus den Berichten zusammensuchen, was bei den auch hier oft mehrere Seiten umfassenden Absätzen nicht ganz einfach ist. Einen Fremdkörper in diesem Kapitel bildet der Bericht über Panama, dessen Entwicklung eher im Zusammenhang mit Kolumbien gesehen werden muss ${ }^{7}$.

Das kurze 4. Kapitel über die Karibik beschränkt sich auf die spanischen Großen Antillen (zu Lateinamerika würde auch Haiti gehören, das freilich kaum geeigneten Stoff geboten hätte $^{8}$ ). Neben der Dominikanischen Republik werden hier Kuba und Puerto Rico behandelt, die beide bis zum Ende des 19. Jahrhunderts dem spanischen Kolonialreich angehörten. Die verfassungsrechtliche Entwicklung unter der spanischen Herrschaft und weiter bis zur Revolution von 1959 wird bei Kuba ausführlich beleuchtet, für die Gegenwart anstelle der fehlenden gerichtlichen Verfassungskontrolle ein kursorischer Überblick über die Justiz gegeben ${ }^{9}$. Dagegen beginnt für Puerto Rico die Darstellung der Verfassungsgeschichte praktisch im 20. Jahrhundert als Teil der USA. Hierbei wird auf die Vermischung der spanischen und angelsächsischen Rechtstradition besonders hingewiesen, wofür freilich bessere Belege zur Verfügung gestanden hätten ${ }^{10}$. Überhaupt scheint das ganze karibische Kapitel nachträglich eingefügt zu sein, denn keine der darin verwendeten Abkürzungen wird im vorangestellten Verzeichnis aufgelöst ${ }^{11}$.

Das 5. Kapitel umfasst alle südamerikanischen Staaten von A(rgentinien) bis V(enezuela) und enthält ebenfalls materialreiche Einzelberichte, die aber wiederum in keinerlei Zusam-

Instruktiv dazu die Entscheidung des Obersten Gerichtshofs vom 30.7.1992, Gaceta Oficial vom 11.11.1992, S. 48 (49 f.); es handelte sich um einen Fall der präventiven Normenkontrolle, für die sich entgegen der Ansicht des Autors in der Praxis des Gerichtshofs zahlreiche Beispiele finden.

Zur Verfassungsentwicklung in Haiti siehe Schmitz, JöR 42 (1994), 613 ff.; zu den Möglichkeiten der Verfassungskontrolle die skeptischen Bemerkungen von Trouillot, Chronique judiciaire d'Haïti 115 (1990), 6 f., die eine cacophonie constitutionelle beklagt.

Allgemeine Überlegungen zur Verfassungsgerichtsbarkeit finden sich bei Fernández Bulté, Revista cubana de derecho 11 (1996), 51-70.

10

Siehe etwa: Mouchet / Sussini, Derecho hispánico y „common law“ en Puerto Rico, Buenos Aires, 1953; Trías Monge, El sistema judicial de Puerto Rico, Puerto Rico, 1978; Thompson, The Introduction of American Law in the Philippines and Puerto Rico, Fayetteville / London, 1989, $156 \mathrm{ff}$.; Negrón, The Legal System of Puerto Rico, Ann Arbor, 1994; jetzt auch Palmer (Hrsg.), Mixed Jurisdictions Worldwide, Cambridge, 2001, 364 ff. Der Autor zitiert stattdessen einen Aufsatz zum mexikanischen amparo-Verfahren (Fn. 711).

11

Das betrifft etwa Abkürzungen wie B.J., CSJ, D.P.R., S.C.J., S.Ct., U.S. Auch sonst enthält das Abkürzungsverzeichnis manche Auslassungen (während die geläufigen deutschen Kürzel sorgfältig aufgeführt sind). 
menhang miteinander stehen ${ }^{12}$. Dass etwa der recurso extraordinario sowohl in Argentinien wie in Brasilien die Überprüfung letztinstanzlicher Urteile am Maßstab der Verfassung ermöglicht, wird aus der Gliederung nicht deutlich: Im Abschnitt über Argentinien wird dieses Verfahren als ,außerordentliches Rechtsmittel“ über mehrere Seiten ausführlich erörtert $^{13}$, bei Brasilien in wenigen Sätzen als Fall der konkreten Normenkontrolle erwähnt. Dem argentinischen Obersten Gerichtshof ist zudem seit 1990 nach nordamerikanischem Vorbild ein weites Ermessen bei der Annahme solcher Verfahren eingeräumt, was aber aus der Darstellung nicht ersichtlich ist ${ }^{14}$. Bei Chile wird die geschichtliche Entwicklung der konkreten Normenkontrolle durch den Obersten Gerichtshof mittels des recurso de inaplicabilidad ausführlich geschildert, für die Gegenwart aber nur in einem Satz resümiert, ohne auf die neuere Rechtsprechung einzugehen ${ }^{15}$ - danach kann der Gerichtshof jetzt auch von Amts wegen ein Gesetz für verfassungswidrig erklären ${ }^{16}$. Dafür werden die Funktionen und Kompetenzen des chilenischen Verfassungsgerichts eingehend beschrieben, insbesondere die präventive Kontrolle der Staatsverträge und derjenigen Gesetzentwürfe, welche die Verfassung ausführen, auslegen oder ändern (sowie die Überprüfung der verschiedenen Arten von Dekreten), wohingegen die ebenso bestehende Möglichkeit der präventiven Kontrolle einfacher Gesetze in der Gliederung nicht auftaucht ${ }^{17}$. Völlig rätselhaft bleibt für den Leser die kritische Konklusion, wonach der Zugang zum Obersten Gerichtshof „in Sachen Verfassungsbeschwerden“ durch dessen eigene Beschlüsse eingeengt worden sei (S. 290 f.). Gemeint sind offenbar die Beschlüsse von 1977 und 1992 über die prozessualen

Nur gelegentlich wird vergleichend auf die Rechtslage in den anderen Staaten hingewiesen, dann aber mit selbstständigen Belegen und ohne jede Querverweisung (Fn. 825 f., 991, 1125 f., 1145).

Der Autor stützt sich dabei auf der Darstellung der einzelnen Fallgruppen bei Sagües, Elementos de derecho constitucional I, 1993, 251 ff., die auf dessen monumentalem zweibändigen Werk „El recurso extraordinario“ von 1984 beruht; dementsprechend ist die Rechtsprechung nur bis zum Jahre 1990 erfasst. Zum historischen Hintergrund siehe Miller, Judicial Review and Constitutional Stability: A Sociology of the U.S. Model and its Collapse in Argentina, Hastings Int.Comp.L.Rev. 21 (1997/98) 77-176.

Siehe Art. 280 der Bundeszivilprozessordnung (idF durch Gesetz 23.774 von 1990, vom Autor selbst auf S. 215 in anderem Zusammenhang mit falschem Datum zitiert). Völlig schief ist die Darstellung, das Gericht, dessen Entscheidung angefochten wird, könne unzulässige Beschwerden durch einen writ of certiorari zurückweisen (S. 223).

Siehe S. 276-278, 282 zu Art. 80 Verf. (abgedruckt in Fn. 1043 und nochmals in Fn. 1050). Anders als auf S. 277 dargestellt, erfasst der recurso de inaplicabilidad heute nicht die Kontrolle vorkonstitutionellen Rechts, die vielmehr als Auslegungsfrage den Untergerichten obliegt; eingehend dazu Precht Pizarro, Rev.Der.Jur. 84 (1987) I, 87 ff. mit ausführlichen Rechtsprechungsnachweisen.

C.S. 26.6.1996, Gaceta Jurídica 192 (1996), 102; zur Bedeutung der Entscheidung ebd. S. 5 f.

$\mathrm{Zu}$ der in Art. 82 Nr. 2 Verf. ausdrücklich vorgesehenen Kontrolle der proyectos de ley siehe Ribera Neumann, Das chilenische Verfassungsgericht und das deutsche Bundesverfassungsgericht, Ein Rechtsvergleich, 1985, 143 ff., bespr. von Gusy, VRÜ 19 (1986) 243-246. Die Besonderheiten bei der präventiven Kontrolle von Staatsverträgen betont Trib.Const. 24.6.1999, Rev.Der.Jur. 96 (1999) II-6-159. 
Voraussetzungen des recurso de protección - dieses durch Art. 20 der chilenischen Verfassung von 1980 zum Schutz der Grundrechte neu geschaffene Rechtsmittel wird aber im vorangehenden Text überhaupt nicht erörtert ${ }^{18}$ ! Dagegen wird das entsprechende tutelaVerfahren der kolumbianischen Verfassung von 1991 ,als schärfste Waffe im Arsenal des kolumbianischen Verfassungsrechts" auf acht Seiten - in einem einzigen Absatz! - ausführlich behandelt, wobei sich der Autor u.a. auf eine Studie seines Doktorvaters Matthias Herdegen stützen konnte ${ }^{19}$. Von ihm übernimmt er auch die Praxis, die Entscheidungen des Verfassungsgerichts nur nach dem Aktenzeichen, aber ohne Datum und Fundstelle zu zitieren $^{20}$. Selbst die wichtige Entscheidung, durch welche der kolumbianische Oberste Gerichtshof überhaupt die Ausarbeitung der geltenden Verfassung von 1991 ermöglichte, wird nur in allgemeiner Form ohne jede Quelle referiert und ist daher für den Leser unauffindbar ${ }^{21}$.

Nach ihrer Funktion gehören sowohl der chilenische recurso de protección wie das kolumbianische tutela-Verfahren in die spanischamerikanische Tradition der amparo-Beschwerde (dem in Brasilien das Rechtsmittel des mandado de segurança entspricht). Dieser Zusammenhang wird auch vom Autor mittelbar anerkannt, wenn er umgekehrt einen Einfluss der tutela auf die Neuregelung des amparo in Ecuador konstatiert ${ }^{22}$. Nur vorläufigen Rechtsschutz bietet das amparo-Verfahren in Paraguay ,sowie ursprünglich in anderen lateinamerikanischen Rechtsordnungen“ - eine Feststellung, die nirgendwo in der Arbeit näher

Vgl. zum recurso de protección schon Wühler / Mayorga Lorca, ZaöRV 41 (1981), 825, 837 f.; zur Erweiterung des Grundrechtsschutzes durch die Verfassungsreform von 1989 siehe Braatz, JöR 41 (1993), 497, 505 f., 521. Zu den Beschlüssen des Obersten Gerichtshof vom 2.4.1977 und 27.6.1992 kritisch Yañez Ramírez, Revista de Derecho 202 (1997), 121 ff.

Herdegen, La tutela contra privados: instrumento certero para implantar la normatividad en la realidad social, Anuario de Derecho Constitucional Latinoamericano 1997, 183-190. Siehe dazu auch Timmermann, Der Schutz der subjektiven Rechte in der kolumbianischen Verfassung, VRÜ 32 (1999), 31-48.

20

Nützlich wäre dabei ein Hinweis auf die entsprechende Datenbank des Justizministeriums: http://www.minjusticia.gov.co/dir_derecho/jurcc.htm. Stattdessen werden in den Fußnoten lange Verfassungstexte mehrfach wiedergegeben, vgl. Fn. 1177 (mit Fn. 1181), Fn. 1179 (mit Fn. 1239) und Fn. 1180 (mit Fn. 1201, 1205, 1207).

Siehe S. 307 f. Es handelt sich um die Plenarentscheidung vom 9.10.1990, abgedruckt in Derecho Colombiano 62, 403, zum Dekret 1926 von 1990 über die Einberufung der verfassunggebenden Versammlung (im Text irreführend auf 1991 datiert!); näher dazu Samtleben, RabelsZ 56 (1992), $176 \mathrm{f}$. Siehe auch die spätere Entscheidung des Verfassungsgerichts vom 3.3.1992, abgedruckt in Foro colombiano 46, 331, zum Ausschluss der gerichtlichen Überprüfung der Verfassung von 1991.

S. 302; dabei stützt er sich nur auf die Bestimmungen der ecuadorianischen Verfassungstexte von 1996 und 1998, ohne auf die „Ley del Control Constitucional“ von 1997 einzugehen (Registro Oficial vom 2.7.1997, S. 5). Diese war kürzlich selbst Gegenstand einer Verfassungsklage: Trib.Const. 24.11.2000, Registro Oficial vom 28.11.2000, Supl. S. 11. 
konkretisiert wird ${ }^{23}$. Zu den traditionellen Grundrechtsschutzverfahren gehört neben dem amparo ferner das Rechtsinstitut des habeas corpus, das auch hier in einigen Berichten vor, in anderen nach oder gemeinsam mit dem amparo-Verfahren abgehandelt wird. Für Chile, wo das klassische habeas corpus-Verfahren auch als recurso de amparo bezeichnet wird, hat der Verfasser überhaupt auf jede Darstellung verzichtet ${ }^{24}$. Die geschichtlichen Ursprünge des habeas corpus, bereits bei Costa Rica ausführlich beschrieben, werden bei Argentinien nochmals in anderer Form dargeboten ${ }^{25}$. Im Übrigen hätte es gerade bei Argentinien nahe gelegen, das amparo-Verfahren nach dem habeas corpus zu behandeln, da es erst im Anschluss an dessen gesetzliche Regelung von der argentinischen Rechtsprechung entwickelt wurde ${ }^{26}$. Auch das habeas data-Verfahren, in Südamerika zuerst in Brasilien und danach in anderen Ländern eingeführt ${ }^{27}$, wird in mehreren der Berichte eingehend dargestellt. Die entsprechende Reform in Peru wird dagegen vom Autor für ,überflüssig“ erklärt, weil dafür auch ein vernünftig gestaltetes amparo-Verfahren gereicht hätte (S. 364) - was dann gleichfalls für die übrigen Länder gelten müsste.

Der Bericht über Peru bedarf ebenso wie der über Venezuela besonderer Hervorhebung: Die Darstellung des peruanischen Systems der Verfassungsgerichtsbarkeit zeigt beispielhaft, wie ein solches System durch die politischen Kräfte pervertiert werden kann. Nach dem „Selbstputsch“ des früheren Präsidenten Fujimori wurde das Verfassungsgericht zunächst aufgelöst, nach seiner Wiedererrichtung durch immer stärkere gesetzliche Beschränkungen seiner eigentlichen Funktion beraubt. Der Autor zeichnet ein plastisches, wenn auch nicht in allen Einzelheiten zutreffendes Bild dieser Entwicklung. Die Vorgänge, die im Mai 1997 zur Absetzung von drei der sieben Verfassungsrichter führten, werden nur

Siehe S. 348. Wenig hilfreich ist auch der dortige Hinweis, die Einzelheiten des paraguayischen amparo-Verfahrens seien ,in einem entsprechenden Gesetz geregelt“; gemeint ist die Zivilprozessordnung von 1988, Art. 565 ff., vgl. Silvero Salgueiro, JöR 46 (1998), 609, 623 f. Durch Gesetz 600/95, Registro Oficial 1995, 1393, wurde das Verfahren zur Feststellung der Verfassungswidrigkeit im amparo-Prozess modifiziert und diese allein dem Obersten Gerichtshof zugewiesen; siehe dazu C.S. 29.2.1996, La Ley, Revista jurídica paraguaya 19 (1996), 85.

Siehe dazu im deutschen Schrifttum die Autoren oben Fn. 18; eingehend Nogueira Alcalá, El Habeas Corpus o Recurso de Amparo en Chile, Revista jurídica del Perú 48 (1998) Nr. 15, S. 45-63.

Siehe S. 90 und 231. Eingehend dazu Sharpe, The Law of Habeas Corpus, Oxford, 1976; zur historischen Entwicklung in Lateinamerika jetzt auch García Belaunde, JöR 49 (2001), 513 ff. Vgl. die entsprechende Darstellung S. 223-226; leider fehlt dort ein Hinweis auf die verdienstvolle Arbeit von $v$. Rohr, Der argentinische Amparo-Prozeß unter Berücksichtigung ähnlicher Verfahren in Brasilien, Mexiko und Peru, 1969, bespr. von Evers, VRÜ 2 (1969), 501-503. Siehe dazu auch Reyven, Der Grundrechtsschutz (habeas corpus, recurso de amparo) im argentinischen Recht, VRÜ 3 (1970), 179-193. 
sehr undeutlich beschrieben ${ }^{28}$. Eine abstrakte Normenkontrolle durch das Verfassungsgericht war danach wegen der dafür erforderlichen Mehrheit von sechs Richtern bis zum Sturz Fujimoris und der Wiedereinsetzung der drei Richter im November 2000 praktisch ausgeschlossen $^{29}$ und ist noch immer erheblichen Beschränkungen unterworfen ${ }^{30}$. Ähnliche Einschränkungen erfuhren die traditionellen Rechtsschutzverfahren des habeas corpus und amparo $^{31}$, was aber von der Rechtsprechung z.T. revidiert worden ist ${ }^{32}$. Auch der Bericht über Venezuela hat aktuellen politischen Bezug. Offenbar hatte der Autor diesen Teil schon abgeschlossen $^{33}$, als die jetzige Verfassung zum 30. Dezember 1999 in Kraft gesetzt wurde (ungenau als Verfassung 1999/2000 bezeichnet). Dementsprechend werden hier zunächst eingehend die venezolanische Verfassungsgeschichte seit den Tagen Großkolumbiens ${ }^{34}$ bis zur Verfassung von 1961 und auf deren Grundlage die verschiedenen Möglichkeiten der Verfassungskontrolle sowie der Grundrechtsschutz im amparo-Verfahren behandelt. In einem ausführlichen Anhang berichtet der Autor dann über die Entstehung der neuen von Präsident Hugo Chávez propagierten „bolivarianischen“ Verfassung und skizziert die wesentlichen Änderungen im Bereich der Verfassungsgerichtsbarkeit. Dabei gelingt ihm

Anlass war eine missliebige Entscheidung der drei Richter, in der sie die Wiederwählbarkeit Fujimoris verneinten (siehe S. 350). Die rechtliche Problematik dieser Entscheidung lag in einer Umfunktionierung der abstrakten Normenkontrollklage in eine „diffuse“ Normenkontrolle (ohne qualifizierte Mehrheit), wie der Verf. erst später andeutet (S. 357 Fn. 1344). Eingehend dazu Fernández Segado, Boletín Mexicano de Derecho Comparado 32 (1999), 765, 803 ff.; Carpio Marcos, ebd. 33 (2000), 447, 473 ff.; jetzt auch Sánchez-Moreno, N.Y.U.J.Int.L.Pol. 33 (2000/01), $561,584 \mathrm{ff}$.

Zutreffend S. 350; irreführend die zugehörige Fn. 1307 und S. 361: Die Herabsetzung des Quorums auf vier Richter durch Gesetz 26801 von 1997 (nicht: 1998) betraf gerade nicht das Verfahren der Normenkontrolle.

Die auf S. 360 genannte Klagefrist von sechs Jahren für die abstrakte Normenkontrolle war bereits durch Gesetz 26618 von 1996 auf sechs Monate verkürzt worden.

Dass beide als acciones de garantía auf denselben gesetzlichen Grundlagen beruhen, ist aus der Darstellung S. 361 f. nicht ersichtlich. Entgegen den dortigen Ausführungen kann mit diesen Klagen seit dem Gesetz 25398 von 1992, Art. 10, die Verletzung von Verfahrensvorschriften nicht mehr gerügt werden; vgl. dazu C.S.21.1.2000, Normas Legales 292, A-3. Auch das Dekret-Gesetz Nr. 25433 von 1992, das den vorläufigen Rechtsschutz im amparo-Verfahren erschwert, wird hier nicht erwähnt (sondern auf S. 356).

So sieht zwar die Verfassung von 1993 ein amparo-Verfahren gegen Gesetze nicht mehr vor (S. 361), die Rechtsprechung hat dies aber gleichwohl in bestimmten Fällen zugelassen, vgl. bei Guzmán Napurí, Normas Legales 295 (2000) A-65 ff. Selbst der gesetzliche Ausschluss des amparo-Verfahrens bei Richterentlassungen (S. 356) ist vom Verfassungsgericht für unwirksam erklärt worden: 13.6.1997, Normas Legales 256, A-7; 12.1.2000, ebd. 287, A-27.

Siehe auch seinen gehaltvollen und gut redigierten Beitrag über die „Verfassungsentwicklung in Venezuela“, JöR 46 (1998) 551-578. der Verfassung von 1821 die Möglichkeit der Verfassungsbeschwerde entnommen (S. 308), hier dagegen jede Bedeutung für die Verfassungsgerichtsbarkeit verneint (S. 379). 
eine ebenso informative wie spannende Darstellung des politischen Hintergrundes sowie der ambivalenten Rolle des Obersten Gerichtshofs ${ }^{35}$ in diesem Entwicklungsprozess.

Etwas aus dem Rahmen fällt der Abschnitt über Brasilien, wo seit 1988 der Oberste Gerichtshof neben anderen Funktionen die Rolle des Verfassungsgerichts wahrnimmt ${ }^{36}$. Die brasilianische Verfassung kennt auf der einen Seite neben der konkreten Normenkontrolle verschiedene Verfahren, um die Verfassungswidrigkeit oder auch (seit 1993) die Verfassungsmäßigkeit eines Gesetzes festzustellen. Zur abstrakten Normenkontrolle liegt bereits eine Arbeit in deutscher Sprache vor, die der Autor aber nicht erwähnt ${ }^{37}$. Nunmehr sind diese Verfassungsklagen in einem Gesetz von Ende 1999 näher geregelt, das er wohl nicht mehr berücksichtigen konnte. Eine Wirkung erga omnes ist in Art. $102 \S 2$ Verf. nur für die Entscheidung über die Verfassungsmäßigkeit vorgesehen; die umstrittene Frage, ob dies auch für die Entscheidung über die Verfassungswidrigkeit gilt, ist durch das Gesetz von 1999 positiv entschieden ${ }^{38}$. Auch die Möglichkeit einer präventiven Verfassungskontrolle wird in Brasilien diskutiert, ist aber bislang nur auf der politischen Ebene verwirklicht $^{39}$. Der Verfasser geht darauf nicht ein, obwohl er an anderer Stelle ,zur grundsätzlichen Problematik der vorbeugenden Normenkontrolle" ausdrücklich auf den Abschnitt Brasilien verweist (Fn. 308). Auf der anderen Seite enthält die Verfassung einen bunten Strauß von sonstigen Rechtsschutzverfahren: habeas corpus, habeas data, mandado de injunção, mandado de segurança (individual und coletivo), ação popular und ação civil pública, von denen jedenfalls die drei letzteren nicht nur der Durchsetzung verfassungsrechtlicher Positionen dienen. Neuartig ist vor allem das mandado de injunção, mit dem die rechtliche Regelung verfassungsmäßig verbürgter Recht verlangt werden kann ${ }^{40}$. Zu all diesen Verfahren werden in den Fußnoten ausführlich die entsprechenden Gesetzestexte, z.T. über ganze Seiten zitiert ${ }^{41}$. Störend wirken dabei die vielen Druckfehler, die wohl auf

Auch für dessen Entscheidungen ist leider vielfach keine Fundstelle angegeben.

Zur politischen Bedeutung der brasilianischen Verfassungsrechtsprechung seit 1988 siehe Faro de Castro, Les tribunaux, le droit et la démocratie au Brésil, Revue internationale des sciences sociales 49 (1997), 265-278.

Ferreira Mendes, Die abstrakte Normenkontrolle vor dem Bundesverfassungsgericht und vor dem brasilianischen Supremo Tribunal Federal (Schriften zum öffentlichen Recht, Bd. 607, 1991).

Gesetz Nr. 9.868 vom 10.11.1999, Art. 28. Eine Wirkung erga omnes im Fall der Verfassungswidrigkeit verneint noch Krell, VRÜ 32 (1999), 8 ff. (21); ebenso Lôbo, Revista de Informação Legislativa 106 (1990), 37 ff. (38), der auch in diesem Fall eine Aufhebung durch den Senat gemäß Art. 52 Nr. X Verf. verlangte; anders Ferreira Mendes (vorige Fn.), 195, unter Hinweis auf die frühere Praxis des Obersten Bundesgerichts. Im Text wird diese Streitfrage nicht erwähnt.

Siehe dazu Cavalcante Lobato, Revista de Informação Legislativa 124 (1994), 169 ff. (176).

Siehe zur Praxis Mont'Alverne Barreto Lima, Staat und Justiz in Brasilien, Frankfurt a.M., 1999, $98 \mathrm{ff}$.

Auch hierbei gibt es vielfache Überschneidungen, siehe Fn. 969 (mit Fn. 976, 980, 1005) und Fn. 978 (mit Fn. 984, 989, 1000, 1013). Der in Fn. 946 abgedruckte angebliche Verfassungstext 
der mangelnden Vertrautheit des Autors mit der portugiesischen Orthographie beruhen. Wo gibt es in Brasilien „Haciendas“ (S. 266)? Allerdings enthält gelegentlich auch der deutsche Text eigenartige Wortbildungen, die selbst durch die neuen Rechtschreibungsregeln nicht gedeckt sind.

Das vorliegende Buch ist in der Reihe „Studien und Materialien zur Verfassungsgerichtsbarkeit“ erschienen. Nach seinem ganzen Zuschnitt wird man es wohl eher zu den „Materialien“ rechnen müssen. Mit Fleiß und Ausdauer hat sein Verfasser viel Material zusammengetragen, das jedem nützlich sein kann, der sich mit Themen der Verfassungsgerichtsbarkeit im lateinamerikanischen Rahmen beschäftigt. Eine „Studie“ ist daraus nicht geworden. Das hätte eine gezielte Fragestellung vorausgesetzt, aber wohl auch die Beschränkung auf bestimmte Schwerpunkte erfordert. Gerade für eine wissenschaftliche Dissertation hätte es nahegelegen, an einer Reihe von ausgewählten Ländern die verschiedenen Modelle der Verfassungsgerichtsbarkeit aufzuzeigen und deren Ausstrahlung in den übrigen Ländern in einem kursorischen Vergleich zu überprüfen ${ }^{42}$. Mit seinem deskriptiven Ansatz, das Recht aller Länder in der gleichen Breite darzustellen, hat sich der Autor weit übernommen und damit der Möglichkeit begeben, den Stoff wirklich zu durchdringen und gedanklich zu bewältigen. Weniger hätte daher in diesem Fall mehr sein können.

beruht offenbar auf einer freien Wiedergabe in einer der Quellen des Autors; in Fn. 995 wurde sogar die Originalfußnote 2 aus der Quelle übernommen. 